\title{
On Computing Robust N-Finger Force-Closure Grasps of 3D Objects
}

\author{
Sahar El-Khoury and Anis Sahbani
}

\begin{abstract}
The paper deals with computing frictional forceclosure grasps of 3D objects problem. The key idea of the presented work is the demonstration that wrenches associated to any three non-aligned contact points of $3 \mathrm{D}$ objects form a basis of their corresponding wrench space. This result permits the formulation of a new sufficient force-closure test. Our approach works with general objects, modelled with a set of points, and with any number $n$ of contacts $(n \geq 4)$. A quality criterion is also introduced. A corresponding algorithm for computing robust force-closure grasps has been developed. Its efficiency is confirmed by comparing it to the classical convexhull method [26].
\end{abstract}

\section{INTRODUCTION}

The stability of a grasp is characterized by force-closure property [9], under which arbitrary forces and torques exerted on the grasped object can be balanced by the contact forces applied by the fingers. Salisbury and Roth have showed that a necessary and sufficient condition for force-closure is that the primitive contact wrenches resulted by contact forces at the contact points positively span the entire 6-dimensional wrench space. This condition is equivalent to that the origin of the wrench space lies strictly inside the convex hull of the primitive contact wrenches [12], [14], [13]. After the pioneering works of Salisbury and Roth [10], several forceclosure necessary and sufficient conditions were proposed in the literature, but only few concerns 3D objects due to their complicated geometry and high dimension of the grasp space [1], [2], [8], [11], [24], [7]. These proposed sufficient and necessary conditions test force-closure by solving a set of linear inequalities or by calculating the $Q$ distance. All these methods require considerable computation time. Generating good grasps (according to various criteria) needs a straightforward test of many grasps configurations, yielding an approach with prohibitive time complexity. An exhaustive search for the best k-finger force-closure grasp of an object modelled by $\mathrm{N}$ points would take time in the order of $O\left(N^{k}\right)$. Thus, heuristic approaches were proposed to improve performance [22], [4], [23]. Their aim is to compute as fast as possible many force-closure grasps. They generate several grasp candidates by selecting contacts on the object surface. Then these grasps are filtered with a necessary but not sufficient force-closure tests. Thus, the grasps that pass the filter may or may not be force-closure. In other words the filter reports false positive but not false negative force-closure grasps. The selected grasps are then tested for force-closure. We propose a new sufficient but not necessary force-closure

S. Khoury is with L2E, Université Pierre et Marie Curie, Paris 6, France. s.khouryelisif.jussieu.fr

A. Sahbani is with ISIR-CNRS, Université Pierre et Marie Curie, Paris 6, UMR 7222, F-75005, France. anis.sahbani@upmc.fr test. Thus, grasps that pass the filter ensure necessarily forceclosure. Our heuristic is original in the sense that it permits not only fast computation but also good quality force-closure grasps generation. This is confirmed by comparing it to the classical convex-hull method [26]. The next paragraph details the problem statement and justifies the choices we made for objects modelling and contacts type and number.

\section{Problem Statement}

Our objective is to find as fast as possible force-closure grasps. In order to determine a grasp, information about contact type and number, and local object surface are required.

An important aspect of the proposed approach is that it is adapted to complex 3D objects. Most works in the literature assume some geometrical model of the objects being grasped [1], [2], [24]. Mainly because this allows efficient or analytical formulation for characterizing grasps. For example, algorithms assuming that an object should be modelled with a polyhedron may work acceptably when the polyhedron faces number is low (objects presented are seldom composed of no more than 20 faces [22]). For real world objects generated by sensor information from cameras or laser scanners the number of faces will normally range from $10^{3}-10^{5}$. Thus, the inaccurate object model may yield to unreliable resulting grasps. Our approach assumes no explicit model of the object being grasped. Objects are modelled with a set of points together with their corresponding normals. As for contact type, hard fingers are considered.

The contacts number depends on the type of contact between the fingertip and the object. When considering 3D objects, Somoff [6] and later Lakshminarayana [5] showed that seven frictionless point contacts are necessary for forceclosure. Mishra, Schwartz and Sharir [12] have shown that tweleve fingers are always sufficient for ensuring forceclosure of 3D objects without rotational symmetries. Markenscoff et al. [30] proved that seven contact points are sufficient for force-closure of 3D non-rotationally objects. An object with rotational symmetry does not have a force-closure grasp with frictionless contacts. They have also shown that in presence of friction, four fingers are sufficient in the 3D case for any object. These bounds were lowered by one contact by Mirtich and Canny [15] who assumed rounded finger tips to provide continuity to the contact normals around the boundary of the object. For grasping and manipulation of objects, a low number of contacts is required thus we assume frictional contacts. Since we want an approach that works with any object geometry even ones with rotational symmetry, we are interested in generating at least 4-finger force-closure grasps. Consequently, our approach can be 
stated as follows:

Given a set of $N$ points along with their normals, we have to compute, as fast as possible, $n$-finger $(n \geq 4)$ force-closure grasps assuming hard-finger frictional contacts.

The rest of the paper is organized as follows. Section 3 recalls notations and theorems useful for the comprehension of the following paragraphs. A new sufficient force-closure condition is presented in section 4 . Our quality criterion used to obtain good grasps is described in section 5. Finally, we discuss in section 6 the experimental results and the performance of the algorithm. Section 7 concludes.

\section{PRELIMINARIES}

This section presents definitions, theorems and notations necessary for force-closure test elaboration.

\section{A. Force-Closure Preliminaries}

The stability of a grasp is characterized by force-closure property. This paragraph presents definitions and theorems necessary for our force-closure test elaboration. First, we remind that Salisbury and Roth [10] has showed that a necessary and sufficient condition for force-closure is that the primitive contact wrenches resulted by contact forces at the contact points positively span the entire wrench space.

Proposition 1: For any n-dimensional Euclidean space $\mathrm{E}^{\mathrm{n}}, n+1$ vectors are necessary to positively span $\mathrm{E}^{\mathrm{n}}$. Proof. for a proof, the reader should refer to the relative linear algebra results presented by Goldman and Tucker [18].

Proposition 2: A set of $n+1$ vectors $v_{1}, v_{2}, \ldots, v_{n+1}$ in $R^{n}$ positively span $E^{n}$ if and only if $v_{n+1}$ is a unique linear combination of $v_{i}, i=1, \ldots, n$ and all coefficients are strictly negative. In other words, the $n+1$ vectors positively span $E^{n}$ if and only if $v_{1}, v_{2}, \ldots, v_{n}$ are linearly independent, thus constitute a basis of $E^{n}$, and $v_{n+1}$ is written as:

$$
v_{n+1}=\sum_{i=1}^{n} \alpha_{i} v_{i}, \quad \alpha_{i}<0
$$

Proof. for a proof, the reader should refer to [19].

\section{B. Grassmann Algebra Preliminaries}

As $3 D$ force-closure grasps involve $6 D$ wrench space. With a mere change of mathematical representation, using Grassmann algebra, we prove that wrenches, associated to any three non-aligned contact points of $3 \mathrm{D}$ objects, form a basis of the $6 D$ wrench space.

Plücker coordinates: Let $L$ be a line in the $3 D$ space. Let $u$ be the unit line direction and $P$ a point chosen on $L$. The direction vector along with its cross product with $P$ are known as Plücker coordinates and are denoted by $(u ; P \times u)$. These 6 coordinates represent $L$ in $3 D$ space [20]. Consequently a primitive contact wrench, defined as $w_{i}=$ $\left(f_{i} ; r_{i} \times f_{i}\right)$ can also be seen as a representation of the line of action $L_{f i}$ of the force $f_{i}$ applied at the point $r_{i}$.

Grassmann algebra : Grassmann studied manifold of lines which rank ranges varies from 0 to 6 . The purpose of his study was to find geometric characterization of each variety. We are going to use two main results of this study. For a proof of these results, the reader should refer to [21].

Proposition 3: All lines through one point are of rank 3.

Proposition 4: When all lines meet one line, they are of rank 5.

\section{A SUFFICIENT CONDITION FOR N-FINGER FORCE-CLOSURE GRASPS}

At this point, we showed that a $6 D$ contact wrench can be represented by the line of action of its corresponding force. We use this mapping to prove that wrenches associated to three non-aligned contact points are of rank 6. This result induces the formulation of a sufficient condition for $\mathrm{n}$-finger $(n>3)$ force-closure grasps (proposition 7).

Proposition 5: The 6 lines on the sides of a tetrahedron are independent, and thus form a basis of $R^{6}$ (Fig. 1).

Proof. To deal with lines in $3 D$-space, we need a 4dimensional linear space. For a basis of this space we can either take a point, $O$ and 3 vectors $e_{1}, e_{2}, e_{3}$ or 4 points $\left(p_{0}, p_{1}, p_{2}, p_{3}\right)$. We can relate these by:

$$
p_{1}=O ; p_{2}=O+e_{1} ; p_{3}=O+e_{2} ; p_{4}=O+e_{3}
$$

Any point can be written as a linear combination of these 4 points, for example:

$$
P_{a}=a_{1} p_{1}+a_{2} p_{2}+a_{3} p_{3}+a_{4} p_{4}
$$

where the $a_{i}$ are scalars and the sum of the $a_{i}$ is unity. Lines are represented in Grassmannian terms by exterior products of points. Hence from these 4 independent basis points we can construct 6 independent lines which intersect to form a tetrahedron :

$$
\begin{aligned}
& L_{1}=p_{1} \wedge p_{2} ; L_{2}=p_{1} \wedge p_{3} ; L_{3}=p_{1} \wedge p_{4} \\
& L_{4}=p_{2} \wedge p_{3} ; L_{5}=p_{2} \wedge p_{4} ; L_{6}=p_{3} \wedge p_{4}
\end{aligned}
$$

Any line $L$ is now able to be represented as a linear combination of these 6 basis lines. We can explicitly display this by multiplying out and simplifying the exterior product of two points $P_{a}$ and $P_{b}$ on the chosen line:

$$
\begin{aligned}
& L=P_{a} \wedge P_{b}=\left(a_{1} p_{1}+a_{2} p_{2}+a_{3} p_{3}+a_{4} p_{4}\right) \wedge \\
& \left(b_{1} p_{1}+b_{2} p_{2}+b_{3} p_{3}+b_{4} p_{4}\right)
\end{aligned}
$$

Proposition 6: Wrenches associated to 3 non-aligned contact points are of rank 6.

Proof. Let $p_{1}, p_{2}$ and $p_{3}$ be 3 non-aligned contact points. Consider the friction cone associated to $p_{1}$, presented in Figure 1, called $C P_{1}$. Let $\left\{m_{1}, m_{2}, m_{3}\right\}$ be three points chosen on any 3 non-coplanar lines of this cone. The lines $\left\{l_{1}=p_{1} \wedge m_{1}, l_{2}=p_{1} \wedge m_{2}, l_{3}=p_{1} \wedge m_{3}\right\}$ are of rank 3 (proposition 3 ). Thus any line that passes through $p_{1}$ can be expressed as a linear combination of these 3 lines. Similarly, $\left\{e_{1}, e_{2}, e_{3}\right\}$ and $\left\{h_{1}, h_{2}, h_{3}\right\}$ are associated respectively to the friction cones $C P_{2}, C P_{3}$ at $p_{2}, p_{3}$. In the same manner, $\left\{l_{4}=p_{2} \wedge e_{1}, l_{5}=p_{2} \wedge e_{2}, l_{6}=p_{2} \wedge e_{3}\right\}$ and $\left\{l_{7}=\right.$ $\left.p_{3} \wedge h_{1}, l_{8}=p_{3} \wedge h_{2}, l_{9}=p_{3} \wedge h_{3}\right\}$ are either of rank 3 . Let 


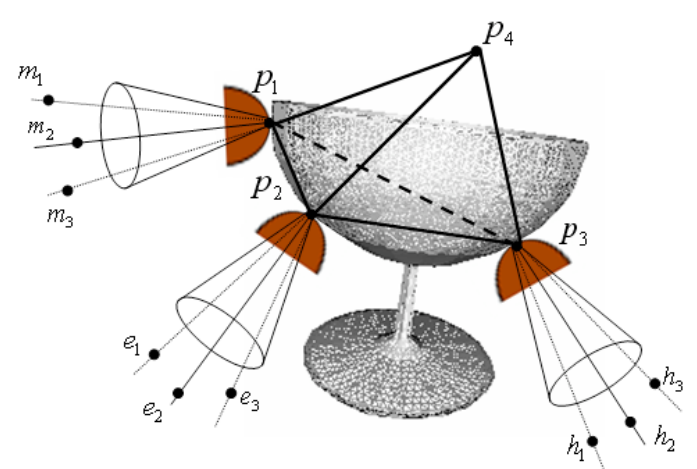

Fig. 1. The wrenches of rank 3 associated to the frictional contact points $p_{1}, p_{2}$ and $p_{3}$.

$p_{4}$ be a point non-coplanar with $p_{1}, p_{2}, p_{3}$, so these 4 points constitute a tetrahedron.

The lines $\left(p_{1} \wedge p_{2}\right),\left(p_{1} \wedge p_{3}\right)$ and $\left(p_{1} \wedge p_{4}\right)$ can be expressed as a linear combination of $\left\{p_{1} \wedge m_{1}, p_{1} \wedge m_{2}, p_{1} \wedge m_{3}\right\}$ since they all pass through $p_{1}$, thus:

$$
p_{1} \wedge p_{j}=\sum_{i=1}^{3} \alpha_{i}^{j}\left(p_{1} \wedge m_{i}\right)=\sum_{i=1}^{3} \alpha_{i}^{j} l_{i}^{j}, \quad j \in 2,3,4
$$

In the same manner, the lines $\left(p_{2} \wedge p_{3}\right)$ and $\left(p_{2} \wedge p_{4}\right)$ can be expressed as a linear combinations of $\left\{p_{2} \wedge e_{1}, p_{2} \wedge e_{2}, p_{2} \wedge e_{3}\right\}$ since they pass through the contact point $p_{2}$. Finally the line $\left(p_{3} \wedge p_{4}\right)$ passes through $p_{3}$ and thus can be expressed as a linear combination of $\left\{p_{3} \wedge h_{1}, p_{3} \wedge h_{2}, p_{3} \wedge h_{3}\right\}$.

Since the lines of the tetrahedron are of rank 6 (proposition 5), they form a basis of $R^{6}$. We showed that the lines of the tetrahedron can be expressed as a linear combination of the 9 lines $l_{i}$. Thus these 9 lines, associated to the 3 friction cones, are also of rank 6 . Consequently, a 6-dimensional basis can be extracted from these 9 lines. We remind the reader that the choice of 3 lines among the $m$ sides of each linearized friction cone is due to the fact that these $m$ lines are of rank 3 (proposition 3).

Proposition 7: Assume that the grasp of $n-1$ non-aligned fingers is not force-closure. Suppose that $\left\{b_{i}\right\}_{i=1 . . k}$ is the $k$-dimensional (where $k=6$ ) basis associated to their corresponding contact wrenches. A sufficient condition for a $n$-finger force-closure grasp is that there exists a contact wrench $\gamma$ such that:

- $\gamma$ is inside the linearized friction cone of the nth finger - $\gamma=\sum_{i=1}^{k} \beta_{i} b_{i}, \beta_{i}<0 \Rightarrow \gamma=B \beta \Rightarrow \beta=B^{-1} \gamma$

where $B=\left[b_{1}, b_{2}, \ldots, b_{k}\right]$ is a $k \times k$ matrix and $\beta=$ $\left[\beta_{1}, \beta_{2}, \ldots, \beta_{k}\right]^{T}$ is a $k \times 1$ strictly negative vector. Thus, a simple multiplication by $B^{-1}$ permits to test if a contact wrench $\gamma$, and consequently the location of the $n t h$ contact point, ensures a force-closure grasp.

Proof. A necessary and sufficient condition for force-closure is that the primitive contact wrenches resulted by contact forces at the contact points positively span the entire k- dimensional wrench space [10]. A set of $k+1$ vectors in $R^{k}$ positively span $E^{k}$ if and only if the $(k+1) t h$ vector is a unique linear combination of the other $k$ vectors and all coefficients are strictly negative (proposition 2). The $k+1$ vectors $\left\{\gamma, b_{1}, b_{2}, . ., b_{k}\right\}$ satisfy these conditions and thus positively $\operatorname{span} R^{k}$.

\section{QUALITY CRITERION OF THE $n-1$ FINGERS LOCATIONS}

At this point, we showed (proposition 7) that to achieve force-closure, we generate randomly locations of $n-1$ nonaligned fingers. A position of the $n t h$ finger is chosen such that an associated contact wrench can be uniquely expressed as a strictly negative linear combination of one of the first generated $n-1$ fingers wrench basis. For generating forceclosure grasps without any quality criterion, the reader should refer to [25], [31]. Our objective is to ensure fast robust force-closure grasps generation. In our case, force-closure grasps fast computation and robustness are strongly linked: one should notice that generating a $n$-finger good grasp will depend on the generation of the first $n-1$ fingers. A good choice of their locations will induce on one hand robust grasps and on the other hand more locations for the nth finger on the object surface guaranteeing force-closure and consequently fast computation. Thus, we need to find a criterion that quantifies a good placement of the $n-1$ first fingers.

Existing force-closure grasps quality criteria permit to select an optimal grasp among a set of stable grasps. Thus, one should generate first several force-closure grasps, compute their corresponding quality to finally choose a good one. A review on the quality measures proposed in the grasp literature can be found in [29]. We propose a method that generates simultaneously force-closure and good quality grasps. The following paragraphs explain the quality criterion we use in the case of a $2 \mathrm{D}$ object and its extension to $3 \mathrm{D}$ objects. In a $2 \mathrm{D}$ case, a wrench basis is represented by three points in the 3D space that constitute with the wrench space origin a tetrahedron. A wrench that ensures force-closure grasp is a wrench that can be uniquely expressed as a strictly negative linear combination of the $3 \mathrm{D}$ basis. Thus, the larger the tetrahedron, the more choices we have for such a wrench. In the following, this idea is detailed more formally.

\section{A. 2D Quality Criterion}

In 2D, a hard finger in contact with an object at a point $x$ exerts a grasp force $f$ with a corresponding torque $\tau=\operatorname{det}(x, f)$. Force and torque are combined into a 3D wrench $w=(f, \tau)$. Thus the wrench space is of rank 3 .

1) $2 D$ force-closure grasps quality: Let $A$ and $B$ be two contact points on the boundary of a planar object. $f_{A 1}, f_{A 2}$ and $f_{B 1}, f_{B 2}$ represent their corresponding friction cones boundaries (Fig. 2). The wrenches associated to these grasp forces are represented respectively by the four 3D points $w_{A 1}, w_{A 2}, w_{B 1}$ and $w_{B 2}$. 
Proposition 8: Wrenches associated to any two contact points of 2D objects form a 3D basis.

Proof. Immediate when one notes that if we select any

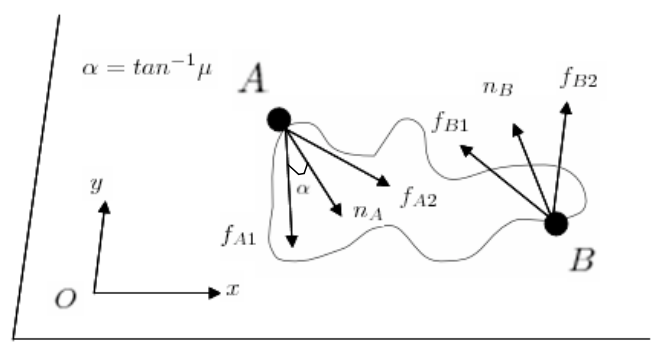

Fig. 2. $f_{A 1}, f_{A 2}$ and $f_{B 1}, f_{B 2}$ represent the $2 D$ friction cones boundaries.

two wrenches, there exists a third one that is not a linear combination of the other two.

Any chosen 3 wrenches from the 4 wrenches associated to the two contact points $A$ and $B$ are of rank 3. Consider for example $w_{A 1}, w_{A 2}$ and $w_{B 1}$, they form a $3 \mathrm{D}$ basis. A sufficient condition for a third finger to ensure 2D force-closure grasp is that its corresponding wrench, $w_{4}$, is a strictly negative linear combination of the 3D basis (proposition 8 ). The convex hull of $w_{A 1}, w_{A 2}, w_{B 1}$ and $w_{4}$ is a tetrahedron $T$. Thus one grasp quality corresponds to the largest ball centered at the origin and inscribed in $T$ [26]. The best quality is obtained when $T$ is a regular one. Consequently, the best locations of the contact points $A$ and $B$ is obtained when the tetrahedron constituted by $w_{A 1}, w_{A 2}, w_{B 1}$ and the origin $O$, approximates a regular one.

2) Tetrahedra quality measure: Different tetrahedron quality measures were proposed in the literature especially in the field of mesh optimization. One of the most used quality is $Q=\frac{V}{h_{\max }^{3}}$ [28], where $V$ is the volume of the tetrahedron and $h_{\max }$ is its maximal edge length. $Q$ is maximal when the corresponding tetrahedron is regular. Using this criterion, the quality of the locations of the two contact points $A$ and $B$ is given by:

$$
Q(A, B)=\max _{i, i=1 . . n b} \frac{V_{i}}{h_{i \max }^{3}}
$$

where $n b$ is the number of tetrahedra constituted by the origin and the wrenches associated to the two contact points. In other words, $n b$ is the number of $3 \mathrm{D}$ basis associated to the contact wrenches, (in $2 D, n b=4$ ).

3) A 2D quality criterion: The reader should keep in mind that we are interested in finding a criterion to the locations of the $n-1$ fingers to ensure robust 3D force-closure grasps. Thus, in the following, we reformulate (1) to be extensible to $3 \mathrm{D}$ grasps. As a matter of fact, the volume $V$ of a tetrahedron can be expressed as the product of a constant, $\delta$, and the determinant of the tetrahedron vertices [27]. Since the origin $O$ is one of the vertices, we obtain:

$$
Q(A, B)=\max _{i, i=1 . . n b} \frac{\delta \cdot \operatorname{det}\left(w_{1}^{i}, w_{2}^{i}, w_{3}^{i}\right)}{h_{i \max }^{3}}
$$

$Q(A, B)$ is maximal when the corresponding tetrahedron is regular. The volume of a regular tetrahedron is $\sqrt{2} a^{3} / 12$, where $a$ is its edges length. For such a tetrahedron, $h_{\text {max }}=a$, thus $Q(A, B)_{\max }$ is $\sqrt{2} / 12$. A normalized criterion will be:

$$
Q(A, B)=\max _{i, i=1 . . n b} \frac{12 . \delta \cdot \operatorname{det}\left(w_{1}^{i}, w_{2}^{i}, w_{3}^{i}\right)}{\sqrt{2} h_{i \max }^{3}}
$$

In order to show the efficiency of the proposed quality criterion, locations of two contact fingers are randomly generated on a 2D object. Using equation (3), the quality of the generated fingers, noted $Q_{f g}$, is computed. All the $2 \mathrm{D}$ object vertices are then tested for force-closure. For all force-closure grasps reported, we calculate the classical grasp quality measure based on the largest ball criterion. The latter is noted $Q_{c l}$. Figure (3) shows the average of $Q_{c l}$ of all force-closure grasps found as a function of $Q_{f g}$ attributed to the fingers, $\left(\operatorname{mean}\left(Q_{c l}\right)=f\left(Q_{f g}\right)\right)$. This figure demonstrates that we are dealing with an increasing function: it means that our criterion and the classical one evolve in the same way. We notice that after a threshold $=0.5$, the force-closure quality obtained is above 0.1 . In other words, when the tetrahedron constituted with the $3 D$ wrench basis and the origin is half-regular, the quality of the force-closure grasps obtained is half-optimal, since the largest ball is of radius $\rho=0.2041$. This value is computed as follows [27], $\rho=3 \cdot \frac{V}{S}=3 \cdot \frac{\sqrt{2}}{12 \cdot \sqrt{3}}=0.2041$, where $V$ is the volume of the tetrahedron of unit length and $S$ the sum of its 4 faces surfaces.

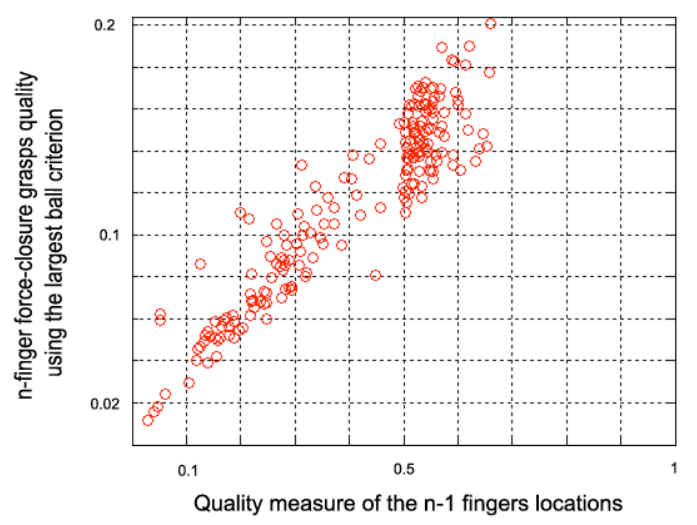

Fig. 3. The n-finger force-closure grasps quality according to the largest ball criterion as a function of the quality measure attributed to the $\mathrm{n}-1$ fingers locations.

\section{B. 3D Quality Criterion}

Dealing with $3 D$ objects grasps involves $6 D$ wrenches. Thus, instead of computing $3 D$ tetrahedra volumes, we are conducted to calculate volumes of $6 D$ hypertetrahedra. Equation (3) introduced in the case of $2 D$ grasps could be extended to $3 D$ grasps as follows (computation of a 6volume hypertetrahedron could be viewed as a determinant 
calculation):

$$
Q(C, D, E)=\max _{i, i=1 . . n b} \frac{\beta \cdot \operatorname{det}\left(w_{1}^{i}, w_{2}^{i}, w_{3}^{i}, w_{4}^{i}, w_{5}^{i}, w_{6}^{i}\right)}{h_{i \max }^{6}}
$$

Where $\left\{w_{j}^{i}\right\}_{j=1 . .6}$ is a $6 D$ wrench basis. Note that $n b$ is the number of $6 D$ basis chosen among the $(n-1) \times m$ wrenches, (corresponding to the $n-1$ fingers). This quality measure is used in the experiments to generate the first $n-1$ contact finger locations on $3 D$ objects.

\section{EXPERIMENTAL RESULTS}

The force-closure test we propose is sufficient but not necessary. In other words, our method reports fault negative results (the method implies no force-closure when it exists). That is due to three reasons. The first one is the linearization of the friction cone. The second is that a point is tested for force-closure with a $6 \mathrm{D}$ basis instead of the convex-hull of all wrenches associated to the $n-1$ fingers. The third is due to testing only the normal wrench, or any positive linear combination of the friction cone sides, associated to the nth finger for force-closure. Thus our approach sacrifices completeness in favor of fast computation. The obvious question is how it competes with a complete method. We choose to use in our experiments the classical complete method based on the construction of a $6 \mathrm{D}$ convex hull [26]. The process involves approximating the contact friction cones as a convex sum of a finite number of force vectors around the boundary of the cone, computing the associated object wrench for each force vector, and then finding the convex hull of this set of wrenches. If the origin is contained within this space, the grasp have force-closure. Otherwise, there exists some set of disturbance wrenches that cannot be resisted by the grasp.

We accomplish tests on a sphere model, represented by its 762 vertices and their respective normal directions. Two experiments are performed in order to show the efficiency of the proposed approach. The first test aims at studying the completeness of the approach. The purpose of the second test is to compare the force-closure grasp computation time of our approach to that of the convex hull. Since these two methods require the cone to be linearized and since all lines through one point are of rank 3 (proposition 3), without loss of generality, we use a 3-sided pyramid to represent a linear model of a cone. The friction coefficient is set to 0.5 , (corresponds to the coefficient between glass and metal). The experiments were run on Pentium Core duo machine with $2 \mathrm{~GB}$ memory and a CPU at $2.13 \mathrm{GHz}$.

\section{A. Completeness Test}

The completeness test aims at computing possible $\mathrm{n}$-finger force-closure grasps on a 3D object with our method and that of the convex-hull. For this purpose, we generate all possible locations for the first three fingers on the sphere. These locations are non-aligned, not ensuring force-closure and which quality is above a threshold. We test then all the remaining vertices for force-closure with all basis associated to the first generated three fingers. Completeness results for different thresholds $0=T h 0<T h 1<T h 2<T h 3<T h 4$
TABLE I

NUMBER OF SOLUTIONS FOR DIFFERENT THRESHOLDS

\begin{tabular}{|l||c|c|c|c|c|}
\hline & Th0 & Th1 & Th2 & Th3 & Th4 \\
\hline \hline Classic & 315 & 349 & 366 & 392 & 419 \\
\hline New & 59 & 82 & 96 & 118 & 149 \\
\hline N/C & $18.73 \%$ & $23.5 \%$ & $26.23 \%$ & $30.10 \%$ & $35.56 \%$ \\
\hline
\end{tabular}

are shown in Table I. The number of solutions presented is averaged by the number of fingers locations combination. Note that Th0 is null and thus presume no constraints on the generation of the first $n-1$ fingers. The latter are thus generated randomly ensuring no minimal quality criterion. We notice that the solutions found by our approach regarding the convex-hull one increases with the threshold. This proves the robustness of the quality criterion proposed. In spite of a good selection of the first three fingers locations, a completeness of only $35 \%$ is obtained. The next paragraph will show that even with a low rate of completeness, i.e with $T h 0$, our approach will generate a more robust force-closure grasp with at least a quarter computation time of the convexhull method.

\section{B. Rapidity Test}

This paragraph measures the computation time for generating one $n$-finger force-closure grasp. Completeness procedure involves testing the nth finger with all $6 \mathrm{D}$ wrench basis associated to the first generated n-1 fingers. Rapidity procedure test force-closure with only one 6D basis chosen according to the introduced quality criterion. The algorithm below details the different corresponding steps for generating $n-1$ finger force-closure grasps.

Require: - 3D points representing the object

- Linearized friction cone at each point and corresponding wrenches

Ensure: - A $n$ fingers force-closure grasp

1: $\alpha \in[0 ; 1]$

2: $\mathrm{L}=$ Rand_Fingers $(n-1)$

3: L_basis $=$ Find_Basis $\left(L_{\_}\right.$wrenches $)$

4: q_L = quality(L_basis)

5: if $\mathrm{q} \mathrm{L}<$ threshold then

6: $\quad$ Go to step 2

7: end if

8: B_basis $=$ Best_Basis (L_basis)

9: randomly choose $x \in] 0 ; 1[$

10: if $x>\alpha$ then

11: $\quad s \leftarrow 0$

12: while $(s<$ Nbpoints $)$ and (No Force-Closure found) do

$s \leftarrow s+1$

vertex=Rand_Finger(nth point)

Force_Closure (vertex, B_basis or L_wrenches) end while

if No Force-Closure found then

Go to step 2

end if 


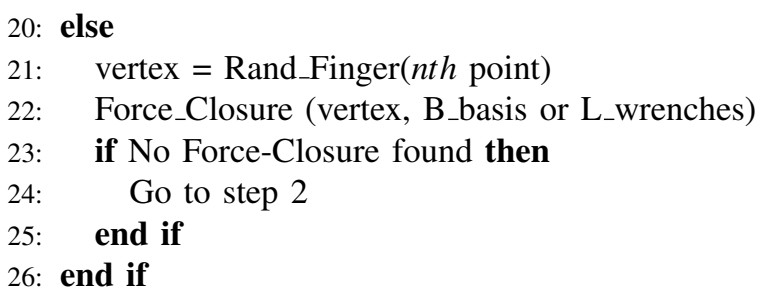

A $n$-finger force-closure grasp may be computed in two different ways. The first one generates randomly locations of $n-1$ fingers non-aligned and not in force-closure on the sphere and then tests till Nbpoints among the object $N$ vertices for force-closure. When no force-closure grasp is found, after testing the Nbpoints, we generate another locations for the first $n-1$ fingers. The second method proceeds also by generating the first $n-1$ fingers but tests then only one randomly generated vertex for force-closure. If the latter does not ensure force-closure, we regenerate another locations for the first $n-1$ fingers. The tuning parameter $\alpha$ will alternate between these two methods. $L$ stands for the randomly generated locations of $n-1$ fingers non-aligned and not in force-closure on the sphere. Since computing a good grasp depends on a good placement of the first $n-1$ fingers as well as the location of the $n t h$ finger, $L$ must ensure a minimal quality criterion. This criterion computation is detailed in the previous paragraph. Once good $n-1$ fingers locations are generated, we determine the best quality basis, B_basis. The value $x$ permits to choose between the two methods. The variable $x$ can take values between 0 and 1 . When $x>0.5$, the second method is privileged. Thus, when no force-closure is obtained when testing a vertex for force-closure with the generated $n-1$ vertices, we privilege regenerating new locations for the $n-1$ vertices on testing another vertex with the same first $n-1$ vertices positions. On the contrary, when $x<0.5$ the first method is privileged. The Force_Closure(vertex,B_basis or L_wrenches) function performs n-finger force closure tests. It takes B_basis or L_wrenches as arguments whether force-closure is tested according to the new or the classic convex-hull method respectively. Experiments were conducted for a 4-finger grasps generation, using different thresholds with the convex-hull and our method. Figure (4) illustrates the influence of the threshold. We remind the reader that Th0 is null and thus presume no constraints on the first $n-1$ generated fingers locations. This graph demonstrates that the better the locations of the first three fingers are chosen, the lower is the computation time of a 4-finger force-closure grasp. The best results are obtained for the threshold $T h 4$. This is normal, because a better choice of the first three fingers increases the number of possible $4 t h$ finger ensuring 4-finger force-closure grasps.

Table (II) compares by varying $\alpha$ our method and that of the convex-hull computation time. The classic line gives in $m s$ the time for computing one 4-finger force-closure grasp according to the previously described algorithm, with the

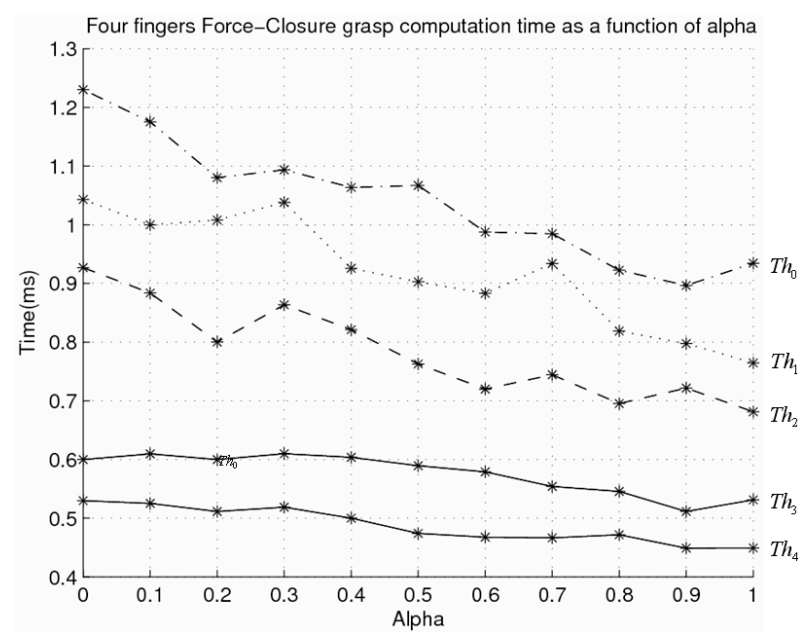

Fig. 4. 4-finger force-closure grasp computation time using our method with thresholds $T h 0<T h 1<T h 2<T h 3<T h 4$

TABLE II

FORCE-CLOSURE GRASP COMPUTATION TIME WITH $T h 0$

\begin{tabular}{|l||c|c|c|c|}
\hline$\alpha$ & 0 & 0.3 & 0.6 & 0.9 \\
\hline \hline Classic & 4.07 & 3.99 & 4.07 & 4.06 \\
\hline New & 1.23 & 1.09 & 0.99 & 0.90 \\
\hline N/C & $30.22 \%$ & $27.32 \%$ & $24.32 \%$ & $22.17 \%$ \\
\hline
\end{tabular}

convex-hull method. The new line does the same when our approach is used. The last line show the ratio of our method computation time to that of the classic method. This ratio varies approximately between $22 \%$ and $30 \%$. Thus in the worst case, when generating the first three fingers randomly without any quality criterion (Th0 is used), our method is about four times faster in finding a force-closure grasp than the convex-hull method. This ratio is about $15 \%$ for $\mathrm{Th} 4$, thus, six times faster than the convex-hull.

Table (III) shows the quality of the force-closure grasps computed with the two approaches. The quality is obtained according to the largest ball criterion [26]. The quality of the force-closure grasps obtained with our approach is approximately twice better than that of the classical method. Thus, although our method is not complete, it finds good solutions for the force-closure problem.

Finally, figure (5) shows the generation of a four-finger force-closure grasp on the grasping part [25] of 3D objects model with different resolutions, a spoon modelled with 629 vertices, a bottle (7360 vertices) and a mug (183534 ver-

TABLE III

FORCE-CLOSURE GRASP GENERATED QUALITY WITH $\alpha=1$

\begin{tabular}{|l||c|c|c|c|c|}
\hline & Th0 & Th1 & Th2 & Th3 & Th4 \\
\hline \hline Classic & 0.0689 & 0.0700 & 0.0731 & 0.0826 & 0.0955 \\
\hline New & 0.1431 & 0.1445 & 0.1458 & 0.1545 & 0.1627 \\
\hline N/C & 2.0769 & 2.0643 & 1.9945 & 1.8705 & 1.7037 \\
\hline
\end{tabular}



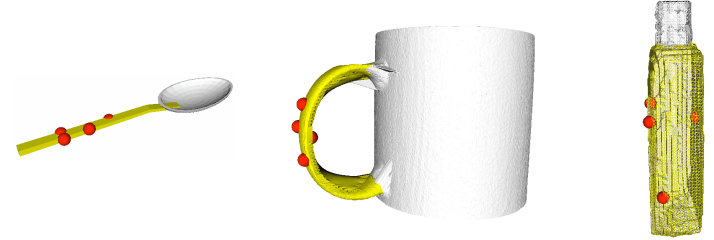

Fig. 5. Force-Closure grasp generated on a synthetic model (spoon), laser scanned model (mug) and a 3D model obtained through a vision system (bottle).

tices). Their corresponding force-closure grasp computation time is respectively $2.59 \mathrm{sec}, 8.87 \mathrm{sec}$ and $4.46 \mathrm{~min}$.

\section{CONCLusions}

Our aim is to compute as fast as possible robust $n$-finger force-closure grasps for a given 3D object. The proposed force-closure sufficient condition is not necessary. Thus, our force-closure test reports fault negative results. However, we showed that our method sacrifices completeness in favor of fast computation which is the real motivation of our work. The completeness test results illustrated in Table I indicate that completeness depends on the quality of the first $n-1$ generated fingers. In the worst case, when the latter are generated randomly, our method finds $18.7 \%$ of the force-closure grasps. This rate may seem low but when considering the quality of the found solutions (table III), one can notice that they are two times better than those found by the classical convex-hull method. Finally, our approach is much faster than the classical method. The computation time depends on the quality of the generated $n-1$ fingers. In the worst case, this time is four times lower than the convex-hull one. This is due to two main reasons:

1) The force-closure test is easy to compute. It is reduced to an inverse matrix calculation.

2) Another main advantage on the convex-hull method is that a construction of a convex-hull is needed whenever a new finger is tested for force-closure with the other $n-1$ fingers. On the other hand, when a basis is associated to the first $n-1$ fingers, it can be used to test all remaining vertices for a n-finger force-closure grasp.

This confirms the efficiency of the proposed method in simplifying computation procedures of force-closure grasps.

\section{ACKNOWLEDGMENTS}

This work is supported by the French ANR (Agence Nationale pour la Recherche) programs under grant No C08527 (ABILIS Project)

\section{REFERENCES}

[1] V.D. Nguyen, Constructing Stable Grasps in 3D, Proc. of Int. Conf. on Robotics and Automation, pp. 234-239, 1987.

[2] J. Ponce, S. Sullivan, J.D. Boissonnat and J.P. Merlet, On Characterizing and Computing Three- and Four-Finger Force-Closure Grasps of Polyhedral Objects, Proc. of Int. Conf. on Robotics and Automation, pp. 821-827, 1993.

[3] P.R. Kraus, V.I. Kumar, and P. Dupont., Analysis of frictional contact models for dynamic symulation., in IEEE Intl. Conf. on Robotics and Automation, 1997.

[4] M. Fischer and G. Hirzinger, Fast planning of precision grasps for $3 D$ objects, Proc. IEEE/RSJ IROS, pp. 120126, 1997.
[5] K. Lakshminarayana, Mechanics of form-closure, Technical Report 78DET-32, ASME, 1978

[6] P. Somoff, Uber gebiete von schraubengeschwindigkeiten eines starren korpers bie verschiedener zahl von stutz achen, Zeitschrift fur Mathematik und Physik, vol. 45, pp. 245-306, 1900.

[7] B. Bounab, D. Sidobre and A. Zaatri, Central Axis Approach for Computing n-Finger Force-Closure Grasps, Proc. IEEE ICRA, 2008.

[8] J.W. Li, H. Liu and H.G. Cai, On Computing Three-finger ForceClosure Grasps of 2D and $3 D$ Objects, IEEE Transactions on Robotics and Automation, 19:(1), 2003.

[9] A. Bicchi, On the closure properties of robotic grasping, Int. J. Robot. Res., vol. 14, no. 4, pp. 319-334, 1995.

[10] J.K. Salisbury and B. Roth, Kinematic and force analysis of articulated hands, ASME J. Mech., Transmissions, Automat., Design, vol. 105, pp. 33-41, 1982.

[11] Y.H. Liu, Qualitative test and force optimization of 3-D frictional form closure grasps using linear programming, IEEE Transactions on Robotics and Automation, vol. 15, no. 1, 1999.

[12] B. Mishra, J.T. Schwartz, and M. Sharir, On the existence and synthesis of multifinger positive grips, Algorithmica, Special Issue: Robotics, vol. 2, pp. 541-558, 1987.

[13] D.J. Montana, The condition for contact grasp stability, in Proc. IEEE Int. Conf. Robot. Automat., pp. 412-417, 1991.

[14] R.M. Murray, Z. Li, and S.S. Sastry, A Mathematical introduction to robotic manipulation, Orlando, FL: CRC, 1994.

[15] B. Mirtich and J. Canny, Easily Computable Optimum Grasps in 2-D and 3-D, Proc. IEEE Int. Conf. Robot. Automat., vol. 1, pp. 739-747, 1994.

[16] D. Ding, Y.H. Liu, and S. Wang, Computing 3D optimal form-closure grasps, Proc. IEEE Int. Conf. Robot. Automat., 2000.

[17] J.C. Trinkle, "On the stability and instantaneous velocity of grasped frictionless objects", IEEE Trans. Robot. Automat., vol. 8, pp. 560572, 1992.

[18] A.J. Goldman and A.W. Tucker, Polyhedral Convex Cones, Princeton University Press, 1956.

[19] R. Wagner, Y. Zhuang and K. Goldberg, Fixturing faceted parts with seven modular struts, IEEE International Symposium on Assembly and Task Planning, USA, 1995.

[20] H. Crapo, A combinatorial perspective on algebraic geometry, Colloquio Int. sulle Teorie Combinatorie, Roma, 1973.

[21] A. Dandurand, The rigidity of compound spatial grid, Structural topology 10, 1984.

[22] Ch. Borst, M. Fischer and G. Hirzinger, Grasping the dice by dicing the grasp, IEEE Int. Conf. on Intelligent Robots and Systems, 2003.

[23] N. Niparnan and A. Sudsang, Positive span of force and torque components of four-fingered three-dimensional force-closure grasps, IEEE Intl. Conf. on Robotics and Automation, 2007.

[24] X. Zhu and J. Wang, Synthesis of Force-Closure Grasps on 3D Objects Based on the $Q$ Distance, IEEE Transcations on robotics and Automation, vol. 19, no. 4, 2003.

[25] S. El-Khoury and A. Sahbani, Handling Objects By Their Handles, IEEE Int. Conf. on Intelligent Robots and Systems, WS - Grasp and Task Learning By Imitation, pp. 58-64, France, 2008.

[26] A.T. Miller and P.K. Allen, Examples of $3 D$ grasp quality computations, Proc. IEEE Intl. Conf. on Robotics and Automation, pp. 12401246, 1999.

[27] P. Robert and A. Roux, Influence of the Shape of the Tetrahedron on the Accuracy of the Estimate of the Current Density, Proceedings of ESA 'START' Conference, France, 1993.

[28] P.D. Zavattieri, G.C. Buscaglia and E.A. Dari, Finite element mesh optimization in three dimensions, 1995.

[29] R. Suarez, M. Roa and J. Cornella, Grasp quality measures, Technical Report, Universitat Politecnica De Catalunya, 2006.

[30] X. Markenscoff and C.H. Papadimitriou, Optimum grip of a polygon, Int. J. Robot. Res., vol. 8:(2), pp. 17-29, 1989.

[31] S. El-Khoury and A. Sahbani, A sufficient condition for computing $n$-finger force-closure grasps of $3 D$ objects, In Proceedings of the 2008 IEEE International Conference on Robotics, Automation and Mechatronics, pp. 791-796, China, 2008. 